\title{
NOUVELLE
}

\section{HLA-DO, régulateur de la réponse immunitaire}

Francis Deshaies, Jacques Thibodeau
Laboratoire d'immunologie

moléculaire, Département

de microbiologie et immunologie,

Faculté de médecine, Université

de Montréal, CP 6128, succursale

Centre-ville, Montréal, Québec H3C

3)7, Canada.

jacques.thibodeau@umontreal.ca

line de surface.

Cela permettrait d'éviter l'activation aléatoire de lymphocytes B potentiellement auto-réactifs [4]. HLA-D0 apparaît donc comme un régulateur naturel de la présentation antigénique. Bien que sa fonction précise demeure inconnue, son rôle inhibiteur de la présentation antigénique laisse présumer qu'une meilleure connaissance de son mode d'action et de sa structure pourrait permettre d'améliorer l'efficacité des vaccins ou encore de combattre les maladies auto-immunes. cules classiques afin de donner naissance à des complexes stables. Plusieurs études soutiennent l'idée d'un modèle selon lequel HLA-DM s'associe aux molécules de classe II instables dans les compartiments lysosomaux et rompt les liens hydrogènes de la liaison de la partie amino-terminale des peptides de faible affinité. Faute de HLA-DM, la diversité du répertoire peptidique est très limitée [5]. Quant à HLADO, sa fonction demeure nébuleuse. Ce chaperon est retrouvé principalement chez les cellules $B$ et uniquement en association avec HLA-DM [4]. HLA-DO inhibe le chargement des peptides dans les endosomes précoces et, paradoxalement, son action négative semble favoriser, directement ou indirectement, le chargement de certains peptides [4]. Les règles moléculaires régissant l'activité de HLAD0 restent à définir, mais il a été démontré in vitro que son action inhibitrice ne se manifeste pas à $\mathrm{pH}$ acide, suggérant un rôle différent dans les compartiments tardifs de la voie endocytique. Il a été proposé que DO inhibe la présentation des antigènes internalisés en phase fluide et donc non reconnus par l'immunoglobu-

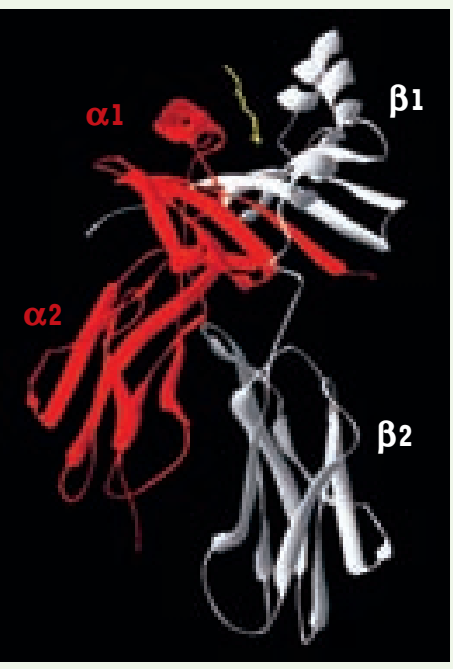

Figure 1. Structure tridimensionnelle de la molécule soluble HLA-DRI liée à un peptide issu du virus influenza. La chaîne $\alpha$ (rouge) et la chaîne $\beta$ (blanche) interagissent avec le peptide antigénique (jaune). Les domaines $\alpha \mathrm{l}$ et $\beta 1$ forment la niche peptidique tandis que les régions $\alpha 2 / \beta 2$ sont ancrées à la membrane par des régions hydrophobes qui sont exclues des formes solubles représentées ici [10]. 
La détermination de la structure tridimensionnelle des molécules de $\mathrm{CMH}-$ I et II par diffraction des rayons $X$ a permis aux groupes de Strominger et Wiley à l'Université Harvard (États-Unis) de définir les bases moléculaires de la fonction de ces molécules, ainsi que la raison d'être du polymorphisme génétique. Depuis la publication de la structure de la molécule HLA-DR en 1993 [2], de nombreuses autres molécules apparentées ont été cristallisées afin de tenter de déterminer, par exemple, une relation possible entre le polymorphisme et les maladies auto-immunes [6].

À l'heure actuelle, les structures tridimensionnelles de HLA-DR et HLA-DQ ont été établies. Du côté des molécules non classiques, la structure de HLA-DM a été déterminée [7]. Quant à la molécule HLA$D 0$, des problèmes techniques ayant trait aux rendements de production des molécules solubles viennent contrecarrer les

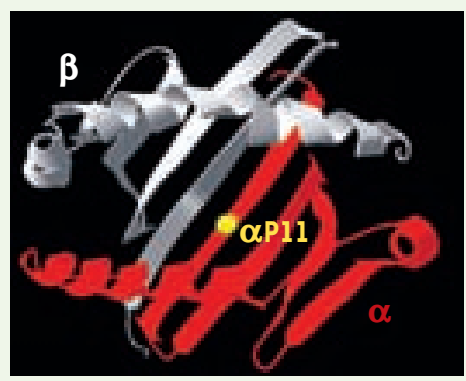

Figure 2. Vue supérieure d'une molécule générique de classe II démontrant la position présumée de l'acide aminé DO $\mathrm{P} 11$ impliqué dans le repliement de la molécule HLA-DO. La chaîne $\alpha$ est représentée en rouge et la chaîne $\beta$ en blanc. La position $\alpha \mathrm{Pll}$ est indiquée par un point jaune. efforts de cristallisation. Cette situation pourrait être due au fait que ce chaperon est entièrement dépendant de HLA-DM pour son repliement dans le réticulum endoplasmique et, par la suite, son transport au-delà de l'appareil de Golgi [4]. Afin de pallier le manque de stabilité de HLA-DO et permettre la production de formes solubles dans les systèmes d'expression des cellules d'insectes, nous avons entrepris de caractériser, à l'échelle moléculaire, les problèmes de repliement des chaînes $\alpha$ et $\beta$. L'étude des structures primaires a révélé que HLA-DO $\alpha$ comporte, tout comme HLA$D Q$, un acide aminé supplémentaire comparativement à la séquence retrouvée chez DR $\alpha$. En effet, DO $\alpha$ comporte l'insertion d'une glycine en position 10 . Cet acide aminé fait preuve d'une très grande liberté dans sa conformation et constitue une protubérance à l'intérieur de la niche peptidique de HLA-DQ, ne modifiant pas la position relative des résidus avoisinants [8]. Cependant, c'est seulement chez HLA-D0 que cette glycine est suivie d'une proline dont la nature rigide laisse deviner des modifications importantes de la structure quaternaire (Figure 2). Nous avons donc entrepris de corriger le défaut potentiel par mutagenèse dirigée sur l'ADNc de DO $\alpha$ en remplaçant la proline par la valine retrouvée sur $D Q \alpha$ [9]. Lorsque cette chaîne $\alpha$ modifiée fut introduite avec la chaîne DO $\beta$ dans des cellules fibroblastiques n'exprimant aucune molécule de classe II, le transport du réticulum endoplasmique vers l'appareil de Golgi a été rapide et similaire à l'hétérodimère de type sauvage en présence de HLA-DM. Le mutant DOPllV, tout comme les molécules de classe II classiques, est donc indépendant de HLA-DM pour sa maturation et son transport. De plus, nous avons démontré in vivo et in vitro qu'en présence de HLA-DM, HLA-DOPIIV est toujours fonctionnel et inhibe le chargement des peptides. Nous croyons que ce variant permettra maintenant la production de formes solubles stables et en quantité suffisante pour parvenir à établir la structure tridimensionnelle de HLA-DO et ainsi mieux comprendre son mode de fonctionnement. $\nabla$

HLA-DO: regulator of the immune response

\section{RÉFÉRENCES}

1. Hunt DF, Michel H, Dickinson TA, et al. Peptides presented to the immune system by murine class II major histocompatibility complex molecule I-A ${ }^{d}$. Science $1992 ; 256: 1817-20$.

2. Brown JH, Jardetzky TS, Gorga JC, et al. Threedimensional structure of the human class II histocompatibility antigen HLA-DR1. Nature 1993 ; $364: 33-9$

3. Marsh SGE, Parham P, Barber LD. The HLA facts book. London: Academic Press, 2000 : 398 p.

4. Karlsson L. DM and DO shape the repertoire of peptide-MHC-class-II complexes. Curr Opin Immunol $2005 ; 17: 65-70$.

5. Miyazaki T, Wolf $P$, Tourne $S$, et al. Mice lacking H2-M complexes, enigmatic elements of the MHC class II peptide-loading pathway. Cell 1996; 84 : 531-41.

6. Housset D, Malissen B. What do TCR-pMHC crystal structures teach us about $\mathrm{MHC}$ restriction and alloreactivity? Trends Immunol 2003 ; 24 : 429-37.

7. Mosyak L, Zaller DM, Wiley DC. The structure of HLA-DM, the peptide exchange catalyst that loads antigen onto class II MHC molecules during antigen presentation. Immunity $1998 ; 9$ : 377-83.

8. Lee KH, Wucherpfennig KW, Wiley DC. Structure of a human insulin peptide-HLA-DQ8 complex and susceptibility to type 1 diabetes. Nat Immunol $2001 ; 2: 501-7$.

9. Deshaies F, Brunet A, Diallo DA, et al. A point mutation in the groove of HLA-DO allows egress from the endoplasmic reticulum independent of HLA-DM. Proc Natl Acad Sci USA 2005 ; 102 : 6443-8.

10. Stern LJ, Brown JH, Jardetzky TS, et al. Crystal structure of the human class II MHC protein HLA-DRI complexed with an inluenza virus peptide. Nature $1994 ; 368: 215-21$.

Retrouvez chaque mois médecine/sciences
sur France-Info dans la chronique « Info-Sciences »
de Marie-Odile Monchicourt, du lundi au mercredi.
france-info.com

